\title{
Existence and uniqueness for dislocation dynamics with nonnegative velocity
}

\author{
O. Alvarez ${ }^{\dagger}$ \\ Lab. Math. R. Salem, Site Colbert, Université de Rouen, 76821 Mont-Saint-Aignan Cedex, France \\ P. CardaliagueT ${ }^{\ddagger}$ \\ Université de Bretagne Occidentale, UFR des Sciences et Techniques, \\ 6 Av. Le Gorgeu, BP 809, 29285 Brest, France \\ AND \\ R. MONnEaU ${ }^{\S}$ \\ CERMICS, Ecole nationale des Ponts et Chaussées, 6 et 8 avenue Blaise Pascal, Cité Descartes, \\ Champs-sur-Marne, 77455 Marne-la-Vallée Cedex 2, France
}

[Received 5 January 2005 and in revised form 1 July 2005]

\begin{abstract}
We study the problem of large time existence of solutions for a mathematical model describing dislocation dynamics in crystals. The mathematical model is a geometric and nonlocal eikonal equation which does not preserve the inclusion. Under the assumption that the dislocation line is expanding, we prove existence and uniqueness of the solution in the framework of discontinuous viscosity solutions. We also show that this solution satisfies some variational properties, which allows us to prove that the energy associated to the dislocation dynamics is nonincreasing.
\end{abstract}

2000 Mathematics Subject Classification: 35F25, 35D05.

Keywords: Dislocation dynamics; eikonal equation; Hamilton-Jacobi equations; discontinuous viscosity solutions; nonlocal equations.

\section{Introduction}

In this paper, we study a simple model for dislocation dynamics. Dislocations are line defects in crystals that can be observed by electron microscopy. The typical length of these dislocation lines in metallic alloys is of the order of $10^{-6} \mathrm{~m}$.

The concept of dislocations in crystals has been introduced and developed in the XXth century, as the main microscopic explanation of the macroscopic plastic behaviour of metallic crystals (see for instance the physical monographs Nabarro [20], Hirth and Lothe [16], or Lardner [18] for a mathematical presentation). Since the beginning of the 90's, the research field of dislocations has enjoyed a new boom based on the increasing power of computers, allowing simulations with a large number of dislocations (see for instance Kubin et al. [17]). This simultaneously motivated new theoretical developments for the modelling of dislocations. Recently Rodney, Le Bouar and Finel introduced in [21] a new model, called the phase field model of dislocations, that we study in this paper.

\footnotetext{
${ }^{\dagger}$ E-mail: olivier.alvarez@wanadoo.fr

E-mail: Pierre.Cardaliaguet@univ-brest.fr

${ }^{\S}$ E-mail: monneau@cermics.enpc.fr
} 
In this model, the dislocation line in the crystal moves in its slip plane with a normal velocity which is proportional to the Peach-Koehler force acting on this line. This force may have two possible contributions: the first one is the self-force created by the elastic field generated by the dislocation line itself; the second one is the force created by everything exterior to the dislocation line, like the exterior stress applied on the material, or the force created by other defects.

Mathematically, a dislocation is formalized by a closed curve $\Gamma(t)$ in $\mathbb{R}^{2}$ moving with a normal velocity $V_{t, x}$ given at each time $t$ and at each point $x \in \Gamma(t)$ by the following nonlocal law:

$$
V_{t, x}=\bar{c}_{0} \star \mathbf{1}_{K(t)}(t, x)+\bar{c}_{1}(t, x) .
$$

In the above equality, $K(t)$ denotes the compact set enclosed by the curve $\Gamma(t)$, the function $\bar{c}_{0}(t, x)$ is a kernel associated to the equations of linearized elasticity and the function $\bar{c}_{1}(t, x)$ describes some external field. The convolution is done in space for $x \in \mathbb{R}^{2}$. Here the term $\bar{c}_{0} \star \mathbf{1}_{K(t)}(t, x)$ corresponds to the part of the velocity created by the self-force, and the term $\bar{c}_{1}(t, x)$ is associated to the exterior forces acting on the dislocation line.

If we set

$$
\rho(t, x)=\mathbf{1}_{K(t)}(x)= \begin{cases}1 & \text { if } x \in K(t), \\ 0 & \text { otherwise }\end{cases}
$$

then equation (1) is equivalent to saying that $\rho$ is a discontinuous viscosity solution of the following nonlocal Hamilon-Jacobi equation (for the definition of discontinuous viscosity solution, see [6], [5]):

$$
\frac{\partial \rho}{\partial t}=\left(\bar{c}_{0} \star \rho(t, \cdot)+\bar{c}_{1}\right)|D \rho|
$$

Such a nonlocal equation has been poorly investigated until now: If $\bar{c}_{0} \geqslant 0$, then the equation satisfies the inclusion principle, and existence and (generic) uniqueness of generalized solutions can be obtained as application of [11]. Unfortunately, for dislocation dynamics, the kernel $\bar{c}_{0}$ has a zero mean, which implies in particular that it changes sign. In [2, 3] short time existence and uniqueness of the solution is proved under the assumption that the initial position of the dislocation is a Lipschitz graph.

In this paper we consider the existence and uniqueness of generalized solutions for an arbitrary time interval, provided that the initial curve is sufficiently smooth and the external field $\bar{c}_{1}$ is large with respect to the kernel $\bar{c}_{0}$, namely we assume that

$$
\bar{c}_{1}(t, x) \geqslant\left\|\bar{c}_{0}(t, \cdot)\right\|_{L^{1}\left(\mathbb{R}^{2}\right)} \quad \forall(t, x) \in[0,+\infty) \times \mathbb{R}^{2} .
$$

This condition ensures that the dislocation is expanding because it implies that, for any Borel subset $K$ of $\mathbb{R}^{2}$, one has

$$
\bar{c}_{0} \star \mathbf{1}_{K}(t, x)+\bar{c}_{1}(t, x) \geqslant \bar{c}_{1}(t, x)-\left\|\bar{c}_{0}(t, \cdot)\right\|_{L^{1}\left(\mathbb{R}^{2}\right)} \geqslant 0 \quad \forall(t, x) \in[0,+\infty) \times \mathbb{R}^{2} .
$$

As for the regularity of the initial curve, we assume that the compact set $K_{0}$ enclosed by this curve satisfies the interior ball condition. This means that there is some $r>0$ such that, for any point $x \in K_{0}$, there is some unit vector $p \in \mathbb{R}^{2}$ with $B(x-r p, r) \subset K_{0}$, where $B(y, r)$ is the closed ball centred at $y$ and of radius $r$ :

$$
\exists r>0, \forall x \in K_{0}, \exists p \in \mathbb{R}^{N},|p|=1 \text { and } B(x-r p, r) \subset K_{0} .
$$


For instance, if $K_{0}$ is the closure of some open bounded set with a $\mathcal{C}^{2}$ boundary, then it satisfies the interior ball condition for some radius $r$.

Under these two assumptions, we prove (in any dimension $N$ ) that the problem of dislocation dynamics has a unique solution $\rho$, and that this solution depends in a Lipschitz way on the initial condition. We also show that this solution $\rho$ is a variational solution, in the sense that

$$
\begin{aligned}
& \int_{\mathbb{R}^{N}} \varphi(t, x) \rho(t, x) \mathrm{d} x-\int_{\mathbb{R}^{N}} \varphi(0, x) \rho(0, x) \mathrm{d} x \\
&=\int_{0}^{t}\left[\int_{\mathbb{R}^{N}} \frac{\partial \varphi}{\partial t}(s, x) \rho(s, x) \mathrm{d} x+\int_{\partial\{\rho(s,)=1\}} \varphi(s, y) c(s, y) \mathrm{d} \mathcal{H}^{N-1}(y)\right] \mathrm{d} s
\end{aligned}
$$

for any $\varphi \in \mathcal{C}^{1}\left([0,+\infty) \times \mathbb{R}^{N}\right)$. As a consequence, we prove that when the data do not depend on time, the energy $E(t)$ naturally associated to the dislocation

$$
E(t)=\int_{\mathbb{R}^{N}}\left(-\frac{1}{2}\left(\bar{c}_{0} \star \rho\right) \rho-\bar{c}_{1} \rho\right)
$$

is nonincreasing:

where $c=\bar{c}_{0} \star \rho+\bar{c}_{1}$.

$$
\frac{\mathrm{d}}{\mathrm{d} t} E(t)=-\int_{\partial\{\rho(t, \cdot)=1\}} c^{2} \mathrm{~d} \mathcal{H}^{N-1},
$$

In order to explain the role played by our two main assumptions (3) and (4), a description of the method of proof is now in order. As in [3] and in Alibaud [1] we use a Banach fixed point argument. We consider the mapping $\Phi$ which associates to any $\rho^{0} \in \mathcal{C}^{0}\left([0, T], L^{1}\left(\mathbb{R}^{N}\right)\right)$, with $0 \leqslant \rho^{0} \leqslant 1$, the unique discontinuous viscosity solution $\rho=\Phi\left(\rho^{0}\right)$ to

$$
\left\{\begin{array}{l}
\frac{\partial \rho}{\partial t}=c_{\rho^{0}}(t, x)|D \rho|, \\
\rho(0, \cdot)=\mathbf{1}_{K_{0}},
\end{array}\right.
$$

where we have set

$$
c_{\rho^{0}}(t, x)=\bar{c}_{0} \star \rho^{0}(t, x)+\bar{c}_{1}(t, x) .
$$

The solution of our problem is clearly a fixed point of $\Phi$. In order to prove that $\Phi$ is a contraction (for a suitable norm, which here turns out to be $\sup _{t \in[0, T]}\|\rho(t, \cdot)\|_{L^{1}\left(\mathbb{R}^{N}\right)}$ ), we are led to combine three types of arguments.

- A representation formula. Since $0 \leqslant \rho^{0} \leqslant 1, c_{\rho^{0}}(t, x)$ is nonnegative and the set $\left\{\Phi\left(\rho^{0}\right)(t, \cdot)=1\right\}$ can be represented as the reachable set of an associated control problem: namely $\left\{\Phi\left(\rho^{0}\right)(t, \cdot)=1\right\}$ is equal to the set of points $z \in \mathbb{R}^{2}$ for which there is some initial position $x_{0} \in K_{0}$ and some measurable map $u:[0, t] \rightarrow \mathbb{R}^{2}$ (the control), with $|u| \leqslant 1$ a.e. in $[0, t]$, and such that the solution to

$$
\left\{\begin{array}{l}
x^{\prime}(s)=c_{\rho^{0}}(t, x) u(s) \\
x(0)=x_{0}
\end{array}\right.
$$

satisfies $x(t)=z$. Then, using the Grownall Lemma, one can easily show that $\Phi$ has the following contraction property: Let $\rho^{0,1}$ and $\rho^{0,2}$ belong to $\mathcal{C}^{0}\left([0, T], L^{1}\left(\mathbb{R}^{N}\right)\right)$ with $0 \leqslant \rho^{0, i} \leqslant 1$ 


$$
\begin{aligned}
& (i=1,2) \text { and } \operatorname{set} K^{1}(t)=\left\{\Phi\left(\rho^{0,1}\right)(t, \cdot)=1\right\} \text { and } K^{2}(t)=\left\{\Phi\left(\rho^{0,2}\right)(t, \cdot)=1\right\} \text {. We have } \\
& \sup _{t \in[0, T]} d^{\mathcal{H}}\left(K^{1}(t), K^{2}(t)\right) \leqslant C T \sup _{t \in[0, T]}\left\|\rho^{0,1}(t, \cdot)-\rho^{0,2}(t, \cdot)\right\|_{L^{1}\left(\mathbb{R}^{2}\right)}
\end{aligned}
$$

where $d^{\mathcal{H}}\left(K^{1}(t), K^{2}(t)\right)$ denotes the Hausdorff distance between the sets $K^{1}(t)$ and $K^{2}(t)$, and $C$ is some given constant (independent of $\rho^{0,1}$ and $\rho^{0,2}$ and $T$ ).

In order to prove that $\Phi$ is indeed a contraction, it remains to show an inequality of the form

$$
\sup _{t \in[0, T]}\left\|\Phi\left(\rho^{0,1}\right)(t, \cdot)-\Phi\left(\rho^{0,2}\right)(t, \cdot)\right\|_{L^{1}\left(\mathbb{R}^{2}\right)} \leqslant C \sup _{t \in[0, T]} d^{\mathcal{H}}\left(K^{1}(t), K^{2}(t)\right) .
$$

This amounts to estimating the volume of the symmetric difference of two sets by their Haudorff distance. In general, such an estimate is hopeless, as simple examples show. It is here that the interior ball condition plays a role.

- Propagation of the interior ball condition. A remarkable property of Hamilton-Jacobi equations of the form (5) is that they preserve the interior ball condition: If the initial set $K_{0}$ satisfies the interior ball condition with radius $r>0$ and if we denote by $\rho(t, x)=\mathbf{1}_{K(t)}(x)$ the solution to (5), then $K(t)$ still satisfies the interior ball condition with some other (but controlled) radius. This result, which has also been noticed in [19], is strongly inspired from [12] and [9]. Let us also point out that [9] contains the much stronger assertion that, when the velocity is positive, the set $K(t)$ develops immediately an interior ball for any compact initial condition $K_{0}$.

- Perimeter and volume estimate of enlarged sets. From the interior ball condition, we can get an inequality of the form (6). Indeed, if a set $K$ satisfies the interior ball condition with some radius $r>0$, then, for any set $K_{1}$, the volume of the difference $K \backslash K_{1}$ can be controlled in terms of the Hausdorff distance between $K$ and $K_{1}$.

This result is a consequence of the following monotonicity formula for the perimeter of a enlarged set: If $K$ is a compact subset of $\mathbb{R}^{N}$, and if we denote by $K+t B$ the set of points which are at a distance less than $t$ of $K$, then the map $t \mapsto \mathcal{H}^{N-1}(\partial(K+t B)) / t^{N-1}$ is nonincreasing.

Let us now explain how this paper is organized: Section 2 is devoted to the monotonicity formula described above and to its applications, among which the fact that the Hausdorff distance controls the volume of the symmetric difference of sets satisfying the interior ball condition. In Section 3 we recall some results on the propagation of the interior ball condition and derive the main estimates needed for proving that the map $\Phi$ has a fixed point. Statement and proof of the existence and uniqueness for (2) are given in Section 4. In Section 5 we give a variational formulation of the problem and show that the energy of the dislocation decreases. We also consider the case of several dynamics. In the appendix we prove the result on the propagation of the interior ball condition.

Let us finally underline that throughout the paper, we work in $\mathbb{R}^{N}$, for $N \geqslant 2$, although the physical problem has a meaning only for $N=2$.

Some notation. We complete this introduction by collecting some notation used throughout the paper. We denote by $|\cdot|$ the euclidean norm of $\mathbb{R}^{N}$, and by $B(x, r)$ the closed ball of radius $r$ centred at the point $x$. If $K$ is a subset of $\mathbb{R}^{N}, d_{K}(x)$ denotes the distance of the point $x$ to the set $K: d_{K}(x)=\inf _{y \in K}|y-x|$. For $r>0$, we denote by $K+r B$ the set of points $x \in \mathbb{R}^{N}$ such that $d_{K}(x) \leqslant r$ and $B=B(0,1)$. Finally, for any function $f$, we denote the gradient of $f$ by $D f$. 


\section{Sets with interior ball condition}

We say that a closed set $K \subset \mathbb{R}^{N}$ satisfies the interior ball condition with radius $r>0$ if, for any point $x \in K$, there is some unit vector $p \in \mathbb{R}^{N}$ with $B(x-r p, r) \subset K$. Then we have the following result (see also [8]):

LEMMA 2.1 If a closed set $K \subset \mathbb{R}^{N}$ satisfies the interior ball condition with radius $r>0$, then there is some closed subset $K_{0}$ of $K$ such that $K=K_{0}+r B$.

From this lemma, it can be easily seen that a closed set $K \subset \mathbb{R}^{N}$ satisfies the interior ball condition with radius $r>0$ if and only if there is a closed set $K_{0} \subset K$ such that $K$ is the set of points $x \in \mathbb{R}^{N}$ with $d_{K_{0}}(x) \leqslant r$. Namely, $K=K_{0}+r B$.

Proof of Lemma 2.1. Set $K_{0}=\left\{x \in K \mid d_{\partial K}(x) \geqslant r\right\}$. Then $K_{0}+r B \subset K$, from the definition of $K_{0}$.

Conversely, let $x \in K$. There is some $p \in \mathbb{R}^{N}$ with $|p|=1$ such that $B(x-r p, r) \subset K$. Hence $x-r p \in K_{0}$ and $d_{K_{0}}(x) \leqslant r$.

In this section we give some estimates of the volume and perimeter of sets satisfying the interior ball condition.

Let us start with an elementary result.

LemMa 2.2 Let $K$ be a closed subset of $\mathbb{R}^{N}$, and let $y_{1}$ and $y_{2}$ be points of $\partial K$ at which $K$ satisfies the interior ball condition with radius $r>0$, that is, there exist unit vectors $p_{1}, p_{2}$ such that $B\left(y_{i}-r p_{i}, r\right) \subset K$ for $i=1,2$. Then

$$
\left\langle p_{1}-p_{2}, y_{1}-y_{2}\right\rangle \leqslant \frac{1}{r}\left|y_{1}-y_{2}\right|^{2} .
$$

Proof. Since $y_{2}$ does not belong to the interior of the ball $B\left(y_{1}-r p_{1}, r\right)$, we have

$$
\left|y_{2}-\left(y_{1}-r p_{1}\right)\right|^{2} \geqslant r^{2}, \quad \text { whence }\left|y_{2}-y_{1}\right|^{2}+2 r\left\langle p_{1}, y_{2}-y_{1}\right\rangle \geqslant 0 \text {. }
$$

In the same way, since $y_{1}$ does not belong to the interior of $B\left(y_{2}-r p_{2}, r\right)$, we have $\left|y_{2}-y_{1}\right|^{2}+$ $2 r\left\langle p_{2}, y_{1}-y_{2}\right\rangle \geqslant 0$. Putting the two inequalities together gives the desired result.

The next lemma plays a crucial role in our study.

LEMmA 2.3 (Monotonicity formula (I)) Let $K$ be a compact subset of $\mathbb{R}^{N}$. Then the function $t \mapsto$ $\mathcal{H}^{N-1}(\partial(K+t B)) / t^{N-1}$ is nonincreasing.

Proof. We start with a preliminary result. Let $0<t_{0}<t_{1}$, let $y_{1}, y_{2}$ belong to $\partial\left(K+t_{1} B\right)$, and $y_{1}^{\prime}, y_{2}^{\prime}$ be projections of $y_{1}, y_{2}$ onto $K+t_{0} B$. We claim that

$$
\left|y_{1}-y_{2}\right| \leqslant \frac{t_{1}}{t_{0}}\left|y_{1}^{\prime}-y_{2}^{\prime}\right| \text {. }
$$

Indeed, let $z_{1}$ and $z_{2}$ be projections of $y_{1}$ and $y_{2}$ respectively onto $K$, and set

$$
p_{1}=\frac{y_{1}-z_{1}}{t_{1}} \quad \text { and } \quad p_{2}=\frac{y_{2}-z_{2}}{t_{1}} .
$$


For $j=1,2$ and $t \in\left[0, t_{1}\right]$, define the maps $y_{j}(t)=z_{j}+t p_{j}$. We note that $y_{j}(t) \in \partial(K+t B)$ for any $t \in\left[0, t_{1}\right]$ and $y_{j}\left(t_{0}\right)=y_{j}^{\prime}$.

Let $\rho(t)=\frac{1}{2}\left|y_{1}(t)-y_{2}(t)\right|^{2}$. Then

$$
\rho^{\prime}(t)=\left\langle y_{1}(t)-y_{2}(t), p_{1}-p_{2}\right\rangle .
$$

Since $y_{j}(t)$ belongs to $\partial(K+t B)$ for $t \in\left[0, t_{1}\right]$ and since the set $K+t B$ satisfies the interior ball condition with radius $t$, from Lemma 2.2 we get

$$
\rho^{\prime}(t) \leqslant \frac{1}{t}\left|y_{1}(t)-y_{2}(t)\right|^{2}=\frac{2}{t} \rho(t) .
$$

Integrating this inequality between $t_{0}$ and $t_{1}$ gives our claim (7).

Next we note that, since $d_{K}$ is a Lipschitz continuous function with compact level sets, the coarea formula states that almost all level sets of $d_{K}$ have finite $\mathcal{H}^{N-1}$ Hausdorff measure. Choose a level $t_{0} \in\left(0, t_{1}\right)$ for which $\mathcal{H}^{N-1}\left(\partial\left(K+t_{0} B\right)\right)<+\infty$.

Let $\epsilon>0$ and $r_{i} \in\left(0, t_{0} \epsilon / 2 t_{1}\right)$ be such that

$$
\partial\left(K+t_{0} B\right) \subset \bigcup_{i=0}^{\infty} A_{i} \quad \text { and } \quad \mathcal{H}^{N-1}\left(\partial\left(K+t_{0} B\right)\right) \geqslant \mathcal{H}^{N-1}\left(B^{N-1}(0,1)\right) \sum_{i=0}^{\infty} r_{i}^{N-1}-\epsilon
$$

for some sets $A_{i}$ of diameter at most $2 r_{i}$, and where $B^{N-1}(0,1)$ is the unit ball of $\mathbb{R}^{N-1}$. We denote by $K_{i}$ the subset of points of $\partial\left(K+t_{1} B\right)$ for which a projection onto $K+t_{0} B$ belongs to $A_{i}$. Then

$$
\partial\left(K+t_{1} B\right) \subset \bigcup_{i=0}^{\infty} K_{i}
$$

We now estimate diam $\left(K_{i}\right)$. Let $y_{1}, y_{2}$ belong to $K_{i}$, and $y_{1}^{\prime}, y_{2}^{\prime}$ be projections of $y_{1}, y_{2}$ onto $K+t_{0} B$ which belong to $A_{i}$. Then from (7), we have

$$
\left|y_{1}-y_{2}\right| \leqslant \frac{t_{1}}{t_{0}}\left|y_{1}^{\prime}-y_{2}^{\prime}\right| \leqslant \frac{t_{1}}{t_{0}} 2 r_{i} .
$$

Hence $\operatorname{diam}\left(K_{i}\right) \leqslant \frac{t_{1}}{t_{0}} 2 r_{i} \leqslant \epsilon$. Therefore

$$
\begin{aligned}
\mathcal{H}_{\epsilon}^{N-1}\left(\partial\left(K+t_{1} B\right)\right) & \leqslant \mathcal{H}^{N-1}\left(B^{N-1}(0,1)\right) \sum_{i=0}^{\infty}\left(\frac{\operatorname{diam}\left(K_{i}\right)}{2}\right)^{N-1} \\
& \leqslant\left(t_{1} / t_{0}\right)^{N-1} \mathcal{H}^{N-1}\left(B^{N-1}(0,1)\right) \sum_{i=0}^{\infty} r_{i}^{N-1} \\
& \leqslant\left(t_{1} / t_{0}\right)^{N-1}\left(\mathcal{H}^{N-1}\left(\partial\left(K+t_{0} B\right)\right)+\epsilon\right) .
\end{aligned}
$$

Letting $\epsilon \rightarrow 0^{+}$gives

$$
\mathcal{H}^{N-1}\left(\partial\left(K+t_{1} B\right)\right) \leqslant\left(\frac{t_{1}}{t_{0}}\right)^{N-1} \mathcal{H}^{N-1}\left(\partial\left(K+t_{0} B\right)\right) .
$$

Hence the $\mathcal{H}^{N-1}$ Hausdorff measure of $\partial(K+t B)$ is finite for any level $t>0$, and the map $\mathcal{H}^{N-1}(\partial(K+t B)) / t^{N-1}$ is nonincreasing. 
For $t>0$, we always have $\partial(K+t B) \subset\left\{d_{K}(x)=t\right\}$, but the inclusion is not an equality in general. This is why we introduce the following variant of the previous monotonicity formula:

LEMMA 2.4 (Monotonicity formula (II)) Let $K$ be a compact subset of $\mathbb{R}^{N}$, and $d_{K}$ the distance function to the set $K$. Then for any $t_{1}>t_{0}>0$, we have

$$
\frac{1}{t_{1}^{N-1}} \mathcal{H}^{N-1}\left(\left\{d_{K}(x)=t_{1}\right\}\right) \leqslant \frac{1}{t_{0}^{N-1}} \mathcal{H}^{N-1}\left(\partial\left(K+t_{0} B\right)\right) .
$$

Proof. The proof is similar to the proof of Lemma 2.3. We only have to replace $\partial\left(K+t_{1} B\right)$ by $\left\{d_{K}(x)=t_{1}\right\}$ everywhere in the proof.

As an application we have the following perimeter estimate for bounded sets which satisfy some interior ball condition.

LEMmA 2.5 Let $0<r<R$. Then, for any compact subset $K$ of $\mathbb{R}^{N}$ such that $K \subset B(0, R)$ and $K$ satisfies the interior ball condition with radius $r$, we have

$$
\mathcal{H}^{N-1}(\partial K) \leqslant N|B| \frac{R^{N}}{r},
$$

where $|B|$ denotes the volume of the unit ball of $\mathbb{R}^{N}$.

Proof. Since $K$ satisfies the interior ball condition with radius $r$, there is some compact set $K_{0}$ such that $K=K_{0}+r B$. Set $K_{t}=K_{0}+t B$. Note that $K_{r}=K$. From Lemma 2.3 we have

$$
\mathcal{H}^{N-1}(\partial K) \leqslant\left(\frac{r}{t}\right)^{N-1} \mathcal{H}^{N-1}\left(\partial K_{t}\right) \quad \forall t \in(0, r] .
$$

Fix $\theta \in(0, r]$. We now apply the coarea formula (see for instance [4]) to the Lipschitz function $d_{K_{\theta}}$ (the distance function from $K_{\theta}$ ): since $\left|D d_{K_{\theta}}\right|=1$ a.e., we have

$$
\begin{aligned}
\left|K \backslash K_{\theta}\right| & =\int_{\theta}^{r} \mathrm{~d} t \int_{\left\{d_{K_{0}}(x)=t\right\}} \mathrm{d} \mathcal{H}^{N-1} \geqslant \int_{\theta}^{r} \mathcal{H}^{N-1}\left(\partial K_{t}\right) \mathrm{d} t \\
& \geqslant \mathcal{H}^{N-1}(\partial K) \int_{\theta}^{r}\left(\frac{t}{r}\right)^{N-1} \mathrm{~d} t \geqslant \frac{\mathcal{H}^{N-1}(\partial K)}{N} \frac{r^{N}-\theta^{N}}{r^{N-1}} .
\end{aligned}
$$

Next we note that $\left|K \backslash K_{\theta}\right| \leqslant|K| \leqslant R^{N}|B|$. Hence

$$
\mathcal{H}^{N-1}(\partial K) \leqslant \frac{N R^{N}|B|}{r-\theta^{N} / r^{N-1}} .
$$

Letting $\theta \rightarrow 0^{+}$gives the result.

Finally, we show that, under the interior ball condition, it is possible to estimate the Hausdorff distance between sets by the difference of their volumes:

LEMMA 2.6 Let $K$ be a compact subset of $\mathbb{R}^{N}$ satisfying the interior ball condition with radius $\sigma>0$. Then for any $r>0$ we have

$$
|(K+r B) \backslash K| \leqslant \frac{\sigma \mathcal{H}^{N-1}(\partial K)}{N}\left(\left(1+\frac{r}{\sigma}\right)^{N}-1\right) .
$$


Proof. Let $K_{0}$ be a compact subset of $\mathbb{R}^{N}$ such that $K_{0}+\sigma B=K$. Then, using Lemma2.4 we get

$$
\begin{aligned}
|(K+r B) \backslash K| & =\int_{\sigma}^{\sigma+r} \mathcal{H}^{N-1}\left(\left\{d_{K_{0}}(x)=t\right\}\right) \mathrm{d} t \\
& \leqslant \mathcal{H}^{N-1}(\partial K) \int_{\sigma}^{\sigma+r}\left(\frac{t}{\sigma}\right)^{N-1} \mathrm{~d} t \leqslant \sigma \frac{\mathcal{H}^{N-1}(\partial K)}{N}\left(\left(1+\frac{r}{\sigma}\right)^{N}-1\right) .
\end{aligned}
$$

\section{Estimates of the reachable set of a controlled system}

In this section we provide our main estimates in order to prove that the map $\Phi$ defined in the introduction is a contraction.

For this, we investigate the propagation of the interior ball for the reachable set of the control system

$$
y^{\prime}(t)=c(t, y(t)) u(t), \quad u(t) \in B(0,1) .
$$

For any initial position $x_{0} \in \mathbb{R}^{N}$ and any measurable control $u:[0,+\infty) \rightarrow B(0,1)$, we denote by $y\left[y_{0}, u\right]$ the solution to $[8]$ with initial position $y\left[y_{0}, u\right](0)=y_{0}$. We denote by $\mathcal{R}(K, t)$ the reachable set at time $t$ when starting from some closed set $K$ :

$$
\mathcal{R}(K, t)=\left\{z \in \mathbb{R}^{N} \mid \exists y_{0} \in K, \exists u:[0,+\infty) \rightarrow B(0,1) \text { measurable, } y\left[y_{0}, u\right](t)=z\right\} .
$$

From now on, we assume that the velocity $c$ has the following regularity properties:

$$
\left\{\begin{array}{l}
\text { (i) } c \text { is nonnegative, continuous, } \\
\text { differentiable with respect to the second variable, } \\
\text { (ii) }|c(t, y)| \leqslant M \quad \forall(t, y) \in \mathbb{R} \times \mathbb{R}^{N}, \\
\text { (iii) }\left|c\left(t, y_{1}\right)-c\left(t, y_{2}\right)\right| \leqslant L_{0}\left|y_{1}-y_{2}\right| \quad \forall\left(t, y_{1}, y_{2}\right) \in \mathbb{R} \times \mathbb{R}^{N} \times \mathbb{R}^{N}, \\
\text { (iv) }\left|D_{x} c\left(t, y_{1}\right)-D_{x} c\left(t, y_{2}\right)\right| \leqslant L_{1}\left|y_{1}-y_{2}\right| \quad \forall\left(t, y_{1}, y_{2}\right) \in \mathbb{R} \times \mathbb{R}^{N} \times \mathbb{R}^{N},
\end{array}\right.
$$

where $M, L_{0}, L_{1} \geqslant 0$ are fixed constants.

Fix also some closed set $K$. When there is no ambiguity, we simply drop $K$ in the notation of the reachable set: $\mathcal{R}(t):=\mathcal{R}(K, t)$. If a point $z$ belongs to the boundary of the set $\mathcal{R}(T)$ for some $T>0$, then there exists a measurable control $b:[0,+\infty) \rightarrow B(0,1)$ and an initial position $y_{0} \in K$ such that $y\left[y_{0}, b\right](T)=z$. It follows from this property that $\mathcal{R}(T)$ is closed. We call such a trajectory $y\left[y_{0}, b\right]$ an extremal trajectory on the time interval $[0, T]$. It is well known that $y\left[y_{0}, b\right](t) \in \partial \mathcal{R}(t)$ for all $t \in[0, T]$.

LEMma 3.1 Assume that the set $K \subset \mathbb{R}^{N}$ is compact and satisfies the interior ball condition with radius $r \in(0,1]$. Then the set $\mathcal{R}(t)$ satisfies the interior ball condition with radius $r e^{-\kappa t}$ for any $t \geqslant 0$, where $\kappa=3 L_{0}+L_{1}$.

Proof. If $z_{0} \in \mathcal{R}(t)$, then there is a time measurable control $b_{0}:[0, t] \rightarrow B(0,1)$ and some $y_{0} \in K$ such that $y\left[y_{0}, b_{0}\right](t)=z_{0}$. We now apply Lemma 6.1 of the appendix to the differential equation with dynamics $f(t, y)=c(t, y) b_{0}(t)$ : the reachable set for this dynamics $f$ starting from $K$ satisfies the interior ball condition with radius $r e^{-\kappa t}$. But this reachable set is contained in $\mathcal{R}(t)$. Hence $\mathcal{R}(t)$ itself satisfies the interior ball condition with radius $r e^{-\kappa t}$. 
In particular, we have:

COROLlary 3.2 Under the assumption of Lemma 3.1, the map

$$
t \mapsto \rho(t)=\mathbf{1}_{\mathcal{R}(t)} \quad \forall t \geqslant 0
$$

is continuous into $L^{1}\left(\mathbb{R}^{N}\right)$.

Proof. From Lemma 3.1, $\mathcal{R}(t)$ satisfies the interior ball condition for any $t \geqslant 0$. So the boundary $\partial \mathcal{R}(t)$ has a zero Lebesgue measure. Since moreover $t \mapsto \mathcal{R}(t)$ is increasing and Hausdorff continuous, the desired result follows.

Let $\mathcal{R}_{i}(t)$ (for $i=1,2$ ) be the reachable set at time $t$ for the controlled system with dynamics

$$
\left\{\begin{array}{l}
y^{\prime}(t)=c_{i}(t, y(t)) b(t), \quad|b(t)| \leqslant 1 \quad \text { a.e. } t \geqslant 0 \\
y(0) \in K_{i} .
\end{array}\right.
$$

We assume that the $K_{i}$ are closed subsets of $\mathbb{R}^{N}$, with $K_{1}, K_{2} \subset B(0, R)$, and satisfy the interior ball condition with radius $r>0$. We also assume that the $c_{i}$ satisfy assumption (9) for $i=1,2$. We fix some $T>0$ and we suppose that

$$
\left\|c_{2}-c_{1}\right\|_{\infty}:=\left\|c_{2}-c_{1}\right\|_{L^{\infty}\left([0, T] \times \mathbb{R}^{N}\right)}<+\infty .
$$

Let $\gamma_{0}>0$ be the Hausdorff distance between $K_{1}$ and $K_{2}$. Recall that $\gamma_{0} \geqslant 0$ is the smallest real number such that

$$
K_{2} \subset K_{1}+\gamma_{0} B \text { and } \quad K_{1} \subset K_{2}+\gamma_{0} B
$$

Our aim is estimate the volume of the symmetric difference $\mathcal{R}_{1}(t) \triangle \mathcal{R}_{2}(t)$.

PROPOSITION 3.3 Under the previous assumptions, for any $t \in[0, T]$ we have

$$
\left|\mathcal{R}_{1}(t) \triangle \mathcal{R}_{2}(t)\right| \leqslant C\left[\gamma_{0}+\left\|c_{1}-c_{2}\right\|_{\infty} t\right]
$$

whenever $\gamma_{0}$ and $\left\|c_{1}-c_{2}\right\|_{\infty}$ are so small that

$$
\left[\gamma_{0}+\left\|c_{1}-c_{2}\right\|_{\infty} T\right] \leqslant r e^{-\left(L_{0}+\kappa\right) T},
$$

where $C=C\left(N, T, M, L_{0}, L_{1}, r, R\right)$ and $\kappa=3 L_{0}+L_{1}$.

To prove the proposition we need a preliminary lemma:

LEMMA 3.4 Under the previous assumptions,

$$
\mathcal{R}_{2}(t) \subset \mathcal{R}_{1}(t)+\gamma(t) B,
$$

where

$$
\gamma(t)=\gamma_{0} e^{L_{0} t}+\left\|c_{1}-c_{2}\right\|_{\infty} \frac{e^{L_{0} t}-1}{L_{0}}
$$


Proof. Let $z \in \mathcal{R}_{2}(t)$. There is a time-measurable control $b:[0, t] \rightarrow B(0,1)$ and a solution $z$ to

$$
\left\{\begin{array}{l}
z^{\prime}(s)=c_{2}(s, z(s)) b(s), \quad \text { a.e. } s \geqslant 0 \\
z(0) \in K_{2}
\end{array}\right.
$$

such that $z(t)=z$. Let $y_{0} \in K_{1}$ be such that $\left|y_{0}-z(0)\right| \leqslant \gamma_{0}$ and let $y$ be the solution to

$$
\left\{\begin{array}{l}
y^{\prime}(s)=c_{1}(s, y(s)) b(s) \\
y(0)=y_{0}
\end{array}\right.
$$

Then

$$
\begin{aligned}
|y(s)-z(s)| & \leqslant\left|y_{0}-z(0)\right|+\int_{0}^{s}\left|c_{1}(\tau, y(\tau))-c_{2}(\tau, z(\tau))\right| \mathrm{d} \tau \\
& \leqslant \gamma_{0}+\left\|c_{1}-c_{2}\right\|_{\infty} s+L_{0} \int_{0}^{s}|y(\tau)-z(\tau)| \mathrm{d} \tau
\end{aligned}
$$

since $c_{1}$ is $L_{0}$-Lipschitz continuous. From the Gronwall Lemma we get

$$
|y(t)-z(t)| \leqslant \gamma(t)
$$

which implies the desired inclusion.

Proof of Proposition 3.3 It is enough to estimate the difference $\left|\mathcal{R}_{2}(t) \backslash \mathcal{R}_{1}(t)\right|$. From Lemma 3.4 we have

$$
\left|\mathcal{R}_{2}(t) \backslash \mathcal{R}_{1}(t)\right| \leqslant\left|\left(\mathcal{R}_{1}(t)+\gamma(t) B\right) \backslash \mathcal{R}_{1}(t)\right| .
$$

By Lemma 3.1 we know that the reachable set $\mathcal{R}_{1}(t)$ satisfies the interior ball condition with radius $\sigma(t)>0$ for any $t \geqslant 0$, where $\sigma(t)=r e^{-\kappa t}$ and $\kappa=3 L_{0}+L_{1}$. Then Lemma 2.6 states that

$$
\left|\left(\mathcal{R}_{1}(t)+\gamma(t) B\right) \backslash \mathcal{R}_{1}(t)\right| \leqslant \frac{\sigma(t) \mathcal{H}^{N-1}\left(\partial \mathcal{R}_{1}(t)\right)}{N}\left(\left(1+\frac{\gamma(t)}{\sigma(t)}\right)^{N}-1\right) .
$$

From assumption 10 we know that

$$
\frac{\gamma(t)}{\sigma(t)}=\left[\gamma_{0} e^{L_{0} t}+\left\|c_{1}-c_{2}\right\|_{\infty} \frac{e^{L_{0} t}-1}{L_{0}}\right] \frac{e^{\kappa t}}{r} \leqslant\left[\gamma_{0}+\left\|c_{1}-c_{2}\right\|_{\infty} T\right] \frac{e^{\left(L_{0}+\kappa\right) T}}{r} \leqslant 1 .
$$

Hence $\left((1+\gamma(t) / \sigma(t))^{N}-1\right) \leqslant N 2^{N-1} \gamma(t) / \sigma(t)$ and we get

$$
\left|\mathcal{R}_{2}(t) \backslash \mathcal{R}_{1}(t)\right| \leqslant C_{N} \mathcal{H}^{N-1}\left(\partial \mathcal{R}_{1}(t)\right) \gamma(t)
$$

for some constant $C_{N}$ which only depends on $N$.

We now note that $\mathcal{R}_{1}(t) \subset B(0, R+M t)$ because $K \subset B(0, R)$ and $\left\|c_{1}\right\|_{\infty} \leqslant M$. Then Lemma 2.5 together with the interior ball estimate gives

$$
\mathcal{H}^{N-1}\left(\partial \mathcal{R}_{1}(t)\right) \leqslant N|B| \frac{(R+M t)^{N} e^{\kappa t}}{r},
$$

from which one gets

$$
\left|\mathcal{R}_{2}(t) \backslash \mathcal{R}_{1}(t)\right| \leqslant C_{N} N|B| \frac{(R+M t)^{N} e^{\kappa t}}{r}\left[\gamma_{0} e^{L_{0} t}+\left\|c_{1}-c_{2}\right\|_{\infty} \frac{e^{L_{0} t}-1}{L_{0}}\right],
$$

yielding the result for a suitable constant $C=C\left(N, T, M, L_{0}, L_{1}, r, R\right)$. 


\section{Application to dislocation dynamics}

We are now ready to investigate the nonlocal equation arising in dislocation dynamics:

$$
\left\{\begin{array}{l}
\frac{\partial \rho}{\partial t}=\left(\bar{c}_{1}+\bar{c}_{0} \star \rho\right)|D \rho| \\
\rho(0, x)=\rho_{0}(x)
\end{array}\right.
$$

where $\rho_{0}(x)=\mathbf{1}_{K_{0}}(x)$.

We assume that $\bar{c}_{0}$ and $\bar{c}_{1}$ are such that

$$
\bar{c}_{1}(t, x) \geqslant\left\|\bar{c}_{0}(t, \cdot)\right\|_{L^{1}\left(\mathbb{R}^{N}\right)} \quad \forall(t, x) \in[0,+\infty) \times \mathbb{R}^{N}
$$

and satisfy for $i=0,1$,

(i) $\bar{c}_{i}$ is uniformly continuous with respect to all the variables and differentiable with respect to the second variable,

(ii) $\left|\bar{c}_{i}(t, y)\right| \leqslant M \quad \forall(t, y) \in \mathbb{R} \times \mathbb{R}^{N}$,

(iii) $\left|\bar{c}_{i}\left(t, y_{1}\right)-\bar{c}_{i}\left(t, y_{2}\right)\right| \leqslant L_{0}\left|y_{1}-y_{2}\right| \quad \forall\left(t, y_{1}, y_{2}\right) \in \mathbb{R} \times \mathbb{R}^{N} \times \mathbb{R}^{N}$,

(iv) $\left|D_{x} \bar{c}_{i}\left(t, y_{1}\right)-D_{x} \bar{c}_{i}\left(t, y_{2}\right)\right| \leqslant L_{1}\left|y_{1}-y_{2}\right| \quad \forall\left(t, y_{1}, y_{2}\right) \in \mathbb{R} \times \mathbb{R}^{N} \times \mathbb{R}^{N}$,

where $M, L_{0}, L_{1} \geqslant 0$ are fixed constants. Recall that assumption $(12)$ implies that

$$
\bar{c}_{1}(t, x)+\bar{c}_{0} \star \mathbf{1}_{K}(t, x) \geqslant 0
$$

for any $(t, x) \in \mathbb{R} \times \mathbb{R}^{N}$ and any Borel measurable set $K$.

In order to explain what we mean by a solution to (11), we have to recall some existence and uniqueness results for the (discontinuous) solutions to Hamilton-Jacobi equations of the form

$$
\left\{\begin{array}{l}
\frac{\partial \rho}{\partial t}=c(t, x)|D \rho|, \\
\rho(0, x)=\mathbf{1}_{K_{0}}(x),
\end{array}\right.
$$

and the link with the reachable set of the control system $(8)$. In the following, we denote by $\rho_{*}$ the lower semicontinuous envelope of some function $\rho$ and by $\rho^{*}$ its upper semicontinuous envelope. We recall that uniqueness for (14) means that all discontinuous solutions have the same lower semicontinuous envelope and the same upper semicontinuous envelope.

LEMMA 4.1 Asume that $c$ is continuous with respect to all variables and satisfies (9), and that $K_{0}$ is equal to the closure of its interior. Then (14) has a unique discontinuous viscosity solution $\rho$. Moreover, for any time $t>0$,

$$
\left\{\rho^{*}(t, \cdot)=1\right\}=\mathcal{R}\left(K_{0}, t\right) \quad \forall t \geqslant 0,
$$

where, as in Section 3, $\mathcal{R}\left(K_{0}, t\right)$ is the reachable set at time $t$ from $K_{0}$ for the controlled system (8).

Proof. The uniqueness result for the geometric evolution equation (14) comes from ([7], Theorem 4.1]). In order to show the link between the level set $\left\{\rho^{*}(t, \cdot)=1\right\}$ and the reachable set $\mathcal{R}\left(K_{0}, t\right)$, let us introduce a new control problem: The value function $u=u(t, x)$ is defined by

$$
u(t, x)=\max _{b} \mathbf{1}_{K_{0}}(y(0))
$$

where $y$ is the solution to the backward differential equation

$$
\left\{\begin{array}{l}
y^{\prime}(s)=c(s, y(s)) b(s) \quad \text { a.e. in }[0, t] \\
y(t)=x
\end{array}\right.
$$


and where the maximum is taken over the measurable maps $b:[0, t] \rightarrow B(0,1)$. By [5] or [6], a routine verification shows that $u$ is a discontinuous viscosity solution to $(14)$, hence it is the unique discontinuous viscosity solution. To complete the proof of the assertion, it suffices to notice that $u$ is upper semicontinuous and that, by definition, $\mathcal{R}\left(K_{0}, t\right)=\left\{x \in \mathbb{R}^{N} \mid u(t, x)=1\right\}$.

Let us now explain what we mean by a viscosity solution to [11] (see also the discussion in [3]).

Definition 4.2 We say that $\rho:[0,+\infty) \times \mathbb{R}^{N} \rightarrow \mathbb{R}$ is a viscosity solution to 11$]$ if $\rho \in$ $\mathcal{C}^{0}\left([0,+\infty), L^{1}\left(\mathbb{R}^{N}\right)\right)$ and $\rho$ is the unique discontinuous viscosity solution to

$$
\left\{\begin{array}{l}
\frac{\partial \rho}{\partial t}=c_{\rho}(t, x)|D \rho|, \\
\rho(0, x)=\rho_{0}(x),
\end{array}\right.
$$

where $c_{\rho}(t, x):=\left(\bar{c}_{1}(t, x)+\left(\bar{c}_{0} \star \rho\right)(t, x)\right)$.

REMARK. Since $\rho \in \mathcal{C}^{0}\left([0,+\infty), L^{1}\left(\mathbb{R}^{N}\right)\right)$ and from assumption $\sqrt[13]{ }$, the function $c_{\rho}$ is continuous with respect to all variables and satisfies $(9)$, with new constants

$$
\begin{aligned}
M^{\prime} & =M \min \left(1+\|\rho\|_{L^{\infty}\left((0, T) ; L^{1}\left(\mathbb{R}^{N}\right)\right)}, 1+\|\rho\|_{L^{\infty}\left((0, T) \times \mathbb{R}^{N}\right)}\right), \\
L_{0}^{\prime} & =L_{0}\left(1+\|\rho\|_{L^{\infty}\left((0, T) ; L^{1}\left(\mathbb{R}^{N}\right)\right)}\right), \quad L_{1}^{\prime}=L_{1}\left(1+\|\rho\|_{L^{\infty}\left((0, T) ; L^{1}\left(\mathbb{R}^{N}\right)\right)}\right) .
\end{aligned}
$$

In the proof of Theorem 4.3 , we will have

$$
\|\rho\|_{L^{\infty}\left((0, T) ; L^{1}\left(\mathbb{R}^{N}\right)\right)} \leqslant 1, \quad\|\rho\|_{L^{\infty}\left((0, T) \times \mathbb{R}^{N}\right)} \leqslant\left|K_{0}\right|+1,
$$

which gives the following choice: $M^{\prime}=2 M, L_{0}^{\prime}=L_{0}\left(2+\left|K_{0}\right|\right), L_{1}^{\prime}=L_{1}\left(2+\left|K_{0}\right|\right)$. From the uniform bound $M^{\prime}=2 M$ on the velocity, we see in particular that if $\operatorname{supp}\left(\rho_{0}\right) \subset B(0, R-\delta)$, the minimal time for the solution to exit the ball $B(0, R)$ is bounded from below by $\delta /(2 M)$, which explains why there will be no blow-up of $\|\rho(t, \cdot)\|_{L^{1}\left(\mathbb{R}^{N}\right)}$ in finite time for this equation.

In the proof of Theorem 4.3 , up to redefining $M, L_{0}, L_{1}$ by $M^{\prime}, L_{0}^{\prime}, L_{1}^{\prime}$, we will keep the notation $M, L_{0}, L_{1}$.

THEOREM 4.3 Assume that the compact set $K_{0}$ satisfies the interior ball condition with radius $r>0$. Then, under assumption (12), the Cauchy problem (11) has a unique discontinuous viscosity solution $\rho$ defined on $[0,+\infty)$.

Moreover, the solution $\rho$ depends in a Lipschitz way on the initial set $K_{0}$ in the following sense: For any $T>0$ and $R>0$, there are constants $\epsilon>0$ and $C>0$ such that, for any compact sets $K_{0}^{i}$ which satisfy the interior ball condition with radius $r$, and such that $K_{0}^{i} \subset B(0, R)$ (for $i=1,2$ ), if we denote by $\rho^{i}$ the unique solution to 11 with initial condition $\mathbf{1}_{K_{0}^{i}}$, then

$$
d^{\mathcal{H}}\left(K_{0}^{1}, K_{0}^{2}\right) \leqslant \epsilon \Rightarrow \sup _{t \in[0, T]}\left\|\rho^{1}(t, \cdot)-\rho^{2}(t, \cdot)\right\|_{L^{1}\left(\mathbb{R}^{N}\right)} \leqslant C d^{\mathcal{H}}\left(K_{0}^{1}, K_{0}^{2}\right),
$$

where $d^{\mathcal{H}}\left(K_{0}^{1}, K_{0}^{2}\right)$ denotes the Hausdorff distance between the sets $K_{0}^{1}$ and $K_{0}^{2}$.

REMARK. With slight modifications of the proofs, it is possible to prove a similar result when $K_{0}$ is the closure of the exterior of a compact set, with $K_{0}$ still satisfying the interior sphere condition.

Proof of Theorem 4.3. We first prove the local existence and uniqueness of the solution. Up to reducing $r$, we can assume that $r \in(0,1]$. Let $R>0$ be such that $K_{0} \subset B(0, R)$, let $T>0$ and set

$$
\mathcal{E}_{T}=\left\{\rho \in \mathcal{C}^{0}\left([0, T], L^{1}\left(\mathbb{R}^{N}\right)\right)\left|\rho(0)=\rho_{0}, 0 \leqslant \rho \leqslant 1, \sup _{t \in[0, T]}\|\rho(t)\|_{1} \leqslant\right| K_{0} \mid+1\right\} .
$$


We fix $T \in(0,1)$ such that

$$
\left[\left\|\bar{c}_{1}\right\|_{\infty}+2\left\|\bar{c}_{0}\right\|_{\infty}\left(\left|K_{0}\right|+1\right)\right] T \frac{e^{\left(L_{0}+\kappa\right) T}}{r} \leqslant 1
$$

where $\kappa=3 L_{0}+L_{1}$, and

$$
C\left[\left\|\bar{c}_{1}\right\|_{\infty}+\left\|\bar{c}_{0}\right\|_{\infty}\left(\left|K_{0}\right|+1\right)\right] T \leqslant 1
$$

where $C=C\left(N, 1, M, L_{0}, L_{1}, r, R\right)$ is the constant given in Proposition 3.3 for $T=1$, and

$$
\theta:=C\left\|\bar{c}_{0}\right\|_{\infty} T<1 .
$$

Note that $T$ only depends - besides the data-on the radius $r>0$ and on the volume $\left|K_{0}\right|$ of $K_{0}$, and is bounded from below by some positive constant as long as $r$ is bounded from below by a positive constant and $\left|K_{0}\right|$ remains bounded.

Let $\Phi$ be the map which associates to any $\rho^{0} \in \mathcal{E}_{T}$ the unique viscosity solution $\rho$ to

$$
\left\{\begin{array}{l}
\frac{\partial \rho}{\partial t}=\left(\bar{c}_{1}+\bar{c}_{0} \star \rho^{0}\right)|D \rho| \\
\rho(0, x)=\rho_{0}(x) .
\end{array}\right.
$$

We first claim that $\Phi\left(\mathcal{E}_{T}\right) \subset \mathcal{E}_{T}$. Indeed, from assumptions (12) and (13), the velocity $c_{1}(t, x)=\bar{c}_{1}(t, x)+\left(\bar{c}_{0} \star \rho^{0}\right)(t, x)$ satisfies assumptions 9 . Corollary 3.2 then states that $\rho \in \mathcal{C}^{0}\left([0, T], L^{1}\left(\mathbb{R}^{N}\right)\right)$.

We want to apply Proposition 3.3 to the velocities $c_{1}$ and $c_{2}=0$ (for which $\rho_{2}(t)=\mathbf{1}_{K_{0}}$ for all $t \geqslant 0)$. For this we first check that $(10)$ holds: Indeed

$$
\begin{aligned}
\left\|c_{1}-c_{2}\right\|_{\infty} T & \leqslant\left(\left\|\bar{c}_{1}\right\|_{\infty}+\left\|\bar{c}_{0}\right\|_{\infty} \sup _{t \in[0, T]}\left\|\rho^{0}(t)\right\|_{1}\right) T \\
& \leqslant\left(\left\|\bar{c}_{1}\right\|_{\infty}+\left\|\bar{c}_{0}\right\|_{\infty}\left(\left|K_{0}\right|+1\right)\right) T \leqslant r e^{-\left(L_{0}+\kappa\right) T}
\end{aligned}
$$

from the choice of $T$ in 16 . Proposition 3.3 then states that (recall that $\rho_{2}(t)=\mathbf{1}_{K_{0}}$ )

$$
\begin{aligned}
\|\rho(t)\|_{1} & =\left\|\rho(t)-\rho_{2}(t)\right\|_{1}+\left|K_{0}\right| \\
& \leqslant C\left[\left\|\bar{c}_{1}\right\|_{\infty}+\left\|\bar{c}_{0}\right\|_{\infty} \sup _{t \in[0, T]}\left\|\rho^{0}(t, \cdot)\right\|_{1}\right] T+\left|K_{0}\right| \leqslant\left|K_{0}\right|+1
\end{aligned}
$$

from (17). Hence $\rho \in \mathcal{E}_{T}$.

Finally, we prove that $\Phi$ is a contraction. Let $\rho_{1}^{0}$ and $\rho_{2}^{0}$ belong to $\mathcal{E}_{T}, c_{1}=\bar{c}_{1}+\bar{c}_{0} \star \rho_{1}^{0}$ and $c_{2}=\bar{c}_{1}+\bar{c}_{0} \star \rho_{2}^{0}, \rho_{1}=\Phi\left(\rho_{1}^{0}\right)$ and $\rho_{2}=\Phi\left(\rho_{2}^{0}\right)$. We first check that $c_{1}$ and $c_{2}$ satisfy condition [10. Indeed,

$$
\begin{aligned}
\left\|c_{1}-c_{2}\right\|_{\infty} T & \leqslant\left\|\bar{c}_{0}\right\|_{\infty} \sup _{t \in[0, T]}\left\|\rho_{1}^{0}(t, \cdot)-\rho_{2}^{0}(t, \cdot)\right\|_{1} T \\
& \leqslant 2\left\|\bar{c}_{0}\right\|_{\infty}\left(\left|K_{0}\right|+1\right) T \leqslant r e^{-\left(L_{0}+\kappa\right) T}
\end{aligned}
$$

from the definition of $\mathcal{E}_{T}$ and the choice of $T$ in 16 . Then using Proposition 3.3 again, we get

$$
\left\|\rho_{1}(t)-\rho_{2}(t)\right\|_{1} \leqslant C\left\|c_{1}-c_{2}\right\|_{\infty} t
$$


which finally gives, from the choice of $T$ in $(18)$,

$$
\left\|\rho_{1}(t)-\rho_{2}(t)\right\|_{1} \leqslant\left(C\left\|\bar{c}_{0}\right\|_{\infty} T\right) \sup _{t \in[0, T]}\left\|\rho_{1}^{0}(t, \cdot)-\rho_{2}^{0}(t, \cdot)\right\|_{1} \leqslant \theta \sup _{t \in[0, T]}\left\|\rho_{1}^{0}(t, \cdot)-\rho_{2}^{0}(t, \cdot)\right\|_{1}
$$

with $\theta<1$.

Since $\Phi$ is a contraction on $\mathcal{E}_{T}$, it has a unique fixed point. So we have proved that the problem has a unique solution $\rho(t, \cdot)=\mathbf{1}_{K(t)}$ at least on the time interval [0,T], where $T$ depends on the volume of $K_{0}$, on $R$ (where $K_{0} \subset B(0, R)$ ) and on the radius of the interior ball $r$ for $K_{0}$. Using Lemma 3.1, we know that the set $K(t)$ satisfies the interior ball condition with radius $r e^{-\kappa t}$, where $\kappa$ depends only on $L_{0}$ and $L_{1}$. Moreover, the volume of $K(t)$ and the radius $R^{\prime}$ such that $K(t) \subset$ $B\left(0, R^{\prime}\right)$ are bounded for bounded times because of the finite speed of propagation. Therefore we can extend the solution in a unique way onto $[0,+\infty)$.

The proof of the Lipschitz continuity of the solution with respect to the initial set is based on arguments similar to those for the local existence and uniqueness, and the use of Proposition 3.3 with $\gamma_{0}=d^{\mathcal{H}}\left(K_{0}^{1}, K_{0}^{2}\right)$.

\section{More on dislocation dynamics}

\subsection{The notion of variational solution}

Our aim is to investigate a notion of weak solution which implies that the natural energy associated to dislocation dynamics is nonincreasing in time. For this reason, these weak solutions will be called variational solutions. In particular, this allows one to prove energy estimates for the generalized evolution. Towards this aim, we first need the following:

Lemma 5.1 Assume that $c=c(t, x)$ satisfies (9) and moreover that

$$
c(t, x)>0 \quad \forall(t, x) \in[0,+\infty) \times \mathbb{R}^{N} .
$$

Let $\mathcal{R}(t)$ be the reachable set (defined in Section 3) at time $t$ starting from some fixed compact set $K \subset \mathbb{R}^{N}$ which satisfies the interior ball condition. Then, for any map $\varphi \in \mathcal{C}^{1}\left([0,+\infty) \times \mathbb{R}^{N}\right)$, the map $t \mapsto \int_{\mathcal{R}(t)} \varphi(t, x) \mathrm{d} x$ is absolutely continuous and

$$
\frac{\mathrm{d}}{\mathrm{d} t} \int_{\mathcal{R}(t)} \varphi(t, x) \mathrm{d} x=\int_{\mathcal{R}(t)} \frac{\partial \varphi}{\partial t}(t, x) \mathrm{d} x+\int_{\partial \mathcal{R}(t)} \varphi(t, y) c(t, y) \mathrm{d} \mathcal{H}^{N-1}(y) .
$$

Proof. Let us introduce the minimal time $\tau: \mathbb{R}^{N} \rightarrow \mathbb{R}$,

$$
\tau(x)=\min \{t \geqslant 0 \mid x \in \mathcal{R}(t)\} \quad \forall x \in \mathbb{R}^{N} .
$$

Under assumptions 99 and $(20)$, the map $\tau$ is locally Lipschitz continuous and satisfies

$$
c(\tau(x), x)|D \tau(x)|=1 \quad \text { for almost all } x \in \mathbb{R}^{N} \backslash K .
$$

In particular assumption (20) implies that, for any $R>0$, there is a constant $\alpha=\alpha(R)>0$ such that

$$
|D \tau(x)| \geqslant \alpha \quad \text { for almost all } x \in B(0, R) \backslash K .
$$

Moreover, $\{\tau \leqslant t\}=\mathcal{R}(t)$ for any $t \geqslant 0$. 
Step 1. Let us first prove that 21 holds for $\varphi=\varphi(x) \in \mathcal{C}_{c}^{\infty}\left(\mathbb{R}^{N}\right)$. From the coarea formula, which we can apply under this form thanks to $(23)$ and the fact that $\varphi$ has a compact support, we have

$$
\int_{\{\tau>t\}} \varphi(x) \mathrm{d} x=\int_{t}^{+\infty} \int_{\{\tau=s\}} \frac{\varphi(y)}{|D \tau(y)|} \mathrm{d} \mathcal{H}^{N-1}(y) \mathrm{d} s .
$$

In order to proceed we need to show that

$$
\mathcal{H}^{N-1}(\{\tau=s\} \backslash \partial \mathcal{R}(s))=0 \text { for almost all } s>0 .
$$

To get this we first note that

$$
\partial^{*}\{\tau>s\} \subset \partial \mathcal{R}(s) \subset\{\tau=s\},
$$

where $\partial^{*}\{\tau>s\}$ denotes the reduced boundary of the set $\{\tau>s\}$. Set $U=\{\tau<t\}$. Using the coarea formula for Lipschitz continuous functions on the one hand and for BV functions (see [14]) on the other hand gives

$$
\int_{U}|D \tau(x)| \mathrm{d} x=\int_{0}^{t} \mathcal{H}^{N-1}(\{\tau=s\})=\int_{0}^{t} \mathcal{H}^{N-1}\left(\partial^{*}\{\tau>s\}\right) .
$$

Hence

$$
\mathcal{H}^{N-1}\left(\{\tau=s\} \backslash \partial^{*}\{\tau>s\}\right)=0 \quad \text { for almost all } s>0,
$$

and therefore 25 holds. Coming back to (24), using first 22 , and then 25 , now gives

$$
\int_{\{\tau>t\}} \varphi(x) \mathrm{d} x=\int_{t}^{+\infty} \int_{\partial \mathcal{R}(s)} \varphi(y) c(s, y) \mathrm{d} \mathcal{H}^{N-1}(y) \mathrm{d} s .
$$

In particular, the map

$$
t \mapsto \int_{\mathcal{R}(t)} \varphi(x) \mathrm{d} x=\int_{\mathbb{R}^{N}} \varphi(x) \mathrm{d} x-\int_{\{\tau>t\}} \varphi(x) \mathrm{d} x
$$

is absolutely continuous and

$$
\frac{\mathrm{d}}{\mathrm{d} t} \int_{\mathcal{R}(t)} \varphi(x) \mathrm{d} x=\int_{\partial \mathcal{R}(t)} \varphi(y) c(t, y) \mathrm{d} \mathcal{H}^{N-1}(y) .
$$

Step 2. We now prove that 21 holds for any $\operatorname{map} \varphi \in \mathcal{C}_{c}^{2}\left([0,+\infty) \times \mathbb{R}^{N}\right)$. For this fix $n \geqslant 1$ and define the partition $\left(t_{k}\right)$ of $[0, t]$ by $t_{k}=k t / n$ for $k=0, \ldots, n$. Then

$$
\int_{\mathcal{R}(t)} \varphi(t, x) \mathrm{d} x-\int_{\mathcal{R}(0)} \varphi(0, x) \mathrm{d} x=\sum_{k=0}^{n-1}\left(\int_{\mathcal{R}\left(t_{k+1}\right)} \varphi\left(t_{k+1}, x\right) \mathrm{d} x-\int_{\mathcal{R}\left(t_{k}\right)} \varphi\left(t_{k}, x\right) \mathrm{d} x\right) .
$$

We have

$$
\begin{aligned}
\int_{\mathcal{R}\left(t_{k+1}\right)} \varphi\left(t_{k+1}, x\right) \mathrm{d} x-\int_{\mathcal{R}\left(t_{k}\right)} \varphi\left(t_{k}, x\right) \mathrm{d} x & \\
= & \int_{\mathcal{R}\left(t_{k+1}\right) \backslash \mathcal{R}\left(t_{k}\right)} \varphi\left(t_{k+1}, x\right) \mathrm{d} x+\frac{t}{n} \int_{\mathcal{R}\left(t_{k}\right)} \frac{\partial \varphi}{\partial t}\left(t_{k}, x\right) \mathrm{d} x+\epsilon\left(t_{k}\right)
\end{aligned}
$$


where

$$
\left|\epsilon\left(t_{k}\right)\right| \leqslant \int_{\mathcal{R}\left(t_{k}\right)}\left|\varphi\left(t_{k+1}, x\right)-\varphi\left(t_{k}, x\right)-\frac{t}{n} \frac{\partial \varphi}{\partial t}\left(t_{k}, x\right)\right| \mathrm{d} x \leqslant \frac{1}{2}\left(\frac{t}{n}\right)^{2}\left\|\frac{\partial^{2} \varphi}{\partial t^{2}}\right\|_{\infty}|\mathcal{R}(t)|
$$

and where, from the first step of the proof,

$$
\int_{\mathcal{R}\left(t_{k+1}\right) \backslash \mathcal{R}\left(t_{k}\right)} \varphi\left(t_{k+1}, x\right) \mathrm{d} x=\int_{t_{k}}^{t_{k+1}} \int_{\partial \mathcal{R}(s)} \varphi\left(t_{k+1}, y\right) c(s, y) \mathrm{d} \mathcal{H}^{N-1}(y) \mathrm{d} s .
$$

Therefore

$$
\begin{aligned}
\int_{\mathcal{R}(t)} \varphi(t, x) \mathrm{d} x-\int_{\mathcal{R}(0)} \varphi(0, x) \mathrm{d} x \\
\quad=\sum_{k=0}^{n-1}\left(\int_{t_{k}}^{t_{k+1}} \int_{\partial \mathcal{R}(s)} \varphi\left(t_{k+1}, y\right) c(s, y) \mathrm{d} \mathcal{H}^{N-1}(y) \mathrm{d} s+\frac{t}{n} \int_{\mathcal{R}\left(t_{k}\right)} \frac{\partial \varphi}{\partial t}\left(t_{k}, x\right) \mathrm{d} x+\epsilon\left(t_{k}\right)\right) .
\end{aligned}
$$

Our aim is to let $n \rightarrow+\infty$ in the above formula. For this, we note that $\mathcal{R}(s)$ is bounded for bounded times and satisfies the interior ball condition with a locally uniform radius (Lemma 3.1). Therefore Lemma 2.5 states that $\mathcal{H}^{N-1}(\partial \mathcal{R}(s))$ is locally uniformly bounded. Thus

$$
\lim _{n} \sum_{k=0}^{n-1} \int_{t_{k}}^{t_{k+1}} \int_{\partial \mathcal{R}(s)} \varphi\left(t_{k+1}, y\right) c(s, y) \mathrm{d} \mathcal{H}^{N-1}(y) \mathrm{d} s=\int_{0}^{t} \int_{\partial \mathcal{R}(s)} \varphi(s, y) c(s, y) \mathrm{d} \mathcal{H}^{N-1}(y) \mathrm{d} s
$$

by the Lebesgue Theorem. We also have

$$
\lim _{n \rightarrow+\infty} \frac{t}{n} \sum_{k=0}^{n-1} \int_{\mathcal{R}\left(t_{k}\right)} \frac{\partial \varphi}{\partial t}\left(t_{k}, x\right) \mathrm{d} x=\int_{0}^{t} \int_{\mathcal{R}(s)} \frac{\partial \varphi}{\partial t}(s, x) \mathrm{d} x
$$

because it is a Riemann sum and the map $s \mapsto \int_{\mathcal{R}(s)} \frac{\partial \varphi}{\partial t}(s, x) \mathrm{d} x$ is continuous since $s \mapsto \mathbf{1}_{\mathcal{R}(s)}$ is continuous into $L^{1}\left(\mathbb{R}^{N}\right)$ from Lemma 3.2. So we have proved that

$$
\begin{aligned}
& \int_{\mathcal{R}(t)} \varphi(t, x) \mathrm{d} x-\int_{\mathcal{R}(0)} \varphi(0, x) \mathrm{d} x \\
&=\int_{0}^{t}\left(\int_{\partial \mathcal{R}(s)} \varphi(s, y) c(s, y) \mathrm{d} \mathcal{H}^{N-1}(y) \mathrm{d} s+\int_{\mathcal{R}(s)} \frac{\partial \varphi}{\partial t}(s, x) \mathrm{d} x\right) \mathrm{d} s,
\end{aligned}
$$

which is the desired result for $\varphi \in \mathcal{C}_{c}^{2}\left([0,+\infty) \times \mathbb{R}^{N}\right)$. We complete the proof of the lemma by density arguments.

A straightforward application of Lemma 5.1 gives:

COROllary 5.2 Assume that $K_{0} \subset \mathbb{R}^{N}$ is compact and satisfies the interior ball condition. Assume that $\bar{c}_{0}$ and $\bar{c}_{1}$ satisfy $(13)$ and that

$$
\bar{c}_{1}(t, x)>\left\|\bar{c}_{0}\right\|_{L^{1}\left(\mathbb{R}^{N}\right)} \quad \forall x \in \mathbb{R}^{N}, \forall t \geqslant 0 .
$$


Let $\rho$ be the unique solution to the dislocation dynamic problem $(11)$. Then $\rho$ also satisfies the following: for any $\varphi \in \mathcal{C}^{1}\left([0,+\infty) \times \mathbb{R}^{N}\right)$,

$$
\begin{aligned}
\int_{\mathbb{R}^{N}} \varphi(t, x) \rho(t, x) \mathrm{d} x-\int_{\mathbb{R}^{N}} \varphi(0, x) \rho(0, x) \mathrm{d} x \\
=\int_{0}^{t}\left[\int_{\mathbb{R}^{N}} \frac{\partial \varphi}{\partial t}(s, x) \rho(s, x) \mathrm{d} x+\int_{\partial\{\rho(s, \cdot)=1\}} \varphi(s, y) c(s, y) \mathrm{d} \mathcal{H}^{N-1}(y)\right] \mathrm{d} s,
\end{aligned}
$$

where $c=\bar{c}_{0} \star \rho+\bar{c}_{1}$.

REMARKS. 1. This equation allows one to define a notion of variational solution for the problem of dislocation dynamics.

2. Equation (26) also holds if $\varphi$ is continuous and such that its time derivative $\partial \varphi / \partial t$ in the sense of distributions is in $L_{\mathrm{loc}}^{1}\left([0,+\infty) \times \mathbb{R}^{N}\right)$.

When the data do not depend on time, namely $\bar{c}_{0}=\bar{c}_{0}(x)$ and $\bar{c}_{1}=\bar{c}_{1}(x)$, and when the kernel $\bar{c}_{0}$ is symmetric, the energy naturally associated to the dislocation is

$$
E(t)=\int_{\mathbb{R}^{N}}\left(-\frac{1}{2}\left(\bar{c}_{0} \star \rho\right) \rho-\bar{c}_{1} \rho\right) .
$$

This energy is nonincreasing:

Proposition 5.3 Under the assumptions and notations of Corollary 5.2 suppose that $\bar{c}_{0}=\bar{c}_{0}(x)$ and $\bar{c}_{1}=\bar{c}_{1}(x)$, and that $\bar{c}_{0}(-x)=\bar{c}_{0}(x)$ for any $x \in \mathbb{R}^{N}$. Then the energy $t \mapsto E(t)$ is locally Lipschitz continuous and

$$
\frac{\mathrm{d}}{\mathrm{d} t} E(t)=-\int_{\partial\{\rho(t, \cdot)=1\}} c^{2} \mathrm{~d} \mathcal{H}^{N-1},
$$

where $c=\bar{c}_{0} \star \rho+\bar{c}_{1}$.

Proof. Let $\varphi(t, x)=\frac{1}{2} \bar{c}_{0} \star \rho+\bar{c}_{1}$. We note that $\varphi$ is continuous and that $t \mapsto \varphi(t, x)$ is absolutely continuous thanks to Corollary 5.2 , with

$$
\frac{\mathrm{d}}{\mathrm{d} t} \varphi(t, x)=\frac{1}{2} \frac{\mathrm{d}}{\mathrm{d} t} \int_{\mathbb{R}^{N}} \bar{c}_{0}(y-x) \rho(t, y) \mathrm{d} y=\frac{1}{2} \int_{\partial\{\rho(t, \cdot)=1\}} \bar{c}_{0}(x-y) c(t, y) \mathrm{d} \mathcal{H}^{N-1}(y) .
$$

Recall (see the proof of Lemma 5.1 that $\mathcal{H}^{N-1}(\partial\{\rho(t, \cdot)=1\})$ is locally bounded. Therefore $t \mapsto \varphi(t, x)$ is locally Lipschitz continuous as also is $t \mapsto E(t)$. So, using Corollary 5.2 again, we have

$$
\frac{\mathrm{d}}{\mathrm{d} t} E(t)=-\int_{\mathbb{R}^{N}} \frac{\partial \varphi}{\partial t} \rho \mathrm{d} x-\int_{\partial\{\rho(t, \cdot)=1\}} \varphi c \mathrm{~d} \mathcal{H}^{N-1},
$$

where

$$
\begin{aligned}
\int_{\mathbb{R}^{N}} \frac{\partial \varphi}{\partial t} \rho \mathrm{d} x & =\frac{1}{2} \int_{\partial\{\rho(t, \cdot)=1\}} \int_{\mathbb{R}^{N}} \bar{c}_{0}(x-y) \rho(t, x) c(t, y) \mathrm{d} x \mathrm{~d} \mathcal{H}^{N-1}(y) \\
& =\frac{1}{2} \int_{\partial\{\rho(t, \cdot)=1\}}\left(\bar{c}_{0} \star \rho\right) c \mathrm{~d} \mathcal{H}^{N-1} .
\end{aligned}
$$

Therefore

$$
\frac{\mathrm{d}}{\mathrm{d} t} E(t)=-\int_{\partial\{\rho(t, \cdot)=1\}}\left(\varphi+\frac{1}{2}\left(\bar{c}_{0} \star \rho\right)\right) c \mathrm{~d} \mathcal{H}^{N-1}=-\int_{\partial\{\rho(t, \cdot)=1\}} c^{2} \mathrm{~d} \mathcal{H}^{N-1} .
$$




\subsection{Dynamics with several dislocations}

Let $M>0$ be an integer. We will assume

$$
\bar{c}_{1}(t, x) \geqslant M\left\|\bar{c}_{0}(t, \cdot)\right\|_{L^{1}\left(\mathbb{R}^{N}\right)} \quad \forall(t, x) \in[0,+\infty) \times \mathbb{R}^{N} .
$$

When we consider the dynamics of $M$ dislocations of the same type (same Burgers vector, and in the same slip plane), it is possible to state a result similar to Theorem 4.3 .

Theorem 5.4 Consider $M$ compact sets $K_{0}^{m}, m=1, \ldots, M$, such that $K_{0}^{1} \supset K_{0}^{2} \supset \cdots \supset K_{0}^{m}$. Assume that each compact $K_{0}^{m}$ satisfies the interior ball condition with radius $r>0$. Then, under assumption (27), the Cauchy problem (11) with initial condition

$$
\rho_{0}(x)=\sum_{m=1}^{M} \mathbf{1}_{K_{0}^{m}}
$$

has a unique discontinuous viscosity solution $\rho$ defined on $[0,+\infty)$.

The proof is an adaptation of the proof of Theorem 4.3 and is left to the reader.

REMARK. If $K_{0}^{m} \supset \supset K_{0}^{m+1}$, then for every time $t>0$, we have $\{\rho \geqslant m\} \supset \supset\{\rho \geqslant m+1\}$. (This is an easy consequence of the representation of each set $\{\rho \geqslant m\}$ as the reachable set for the controlled system $\left[8\right.$ with $c(t, x)=\bar{c}_{0} \star \rho+\bar{c}_{1}$.)

\section{Appendix: Propagation of the interior ball condition}

In this section, we consider a differential equation

$$
y^{\prime}(t)=f(t, y(t)) .
$$

The reachable set for $f$ when starting from an initial closed set $K$ is defined in the usual way and denoted $\mathcal{R}(t)$ as before. Our aim is to show that this reachable set satisfies the interior ball condition provided the initial set does. The computations below are strongly inspired by those of [9].

For this we assume that $f$ enjoys the following regularity:

(i) $f$ is Borel measurable, differentiable with respect to the second variable for almost every $t$

(ii) $\left|f\left(t, y_{1}\right)-f\left(t, y_{2}\right)\right| \leqslant L_{0}\left|y_{1}-y_{2}\right| \quad \forall\left(t, y_{1}, y_{2}\right) \in \mathbb{R} \times \mathbb{R}^{N} \times \mathbb{R}^{N}$,

(iii) $\left|D_{x} f\left(t, y_{1}\right)-D_{x} f\left(t, y_{2}\right)\right| \leqslant L_{1}\left|y_{1}-y_{2}\right| \quad \forall\left(t, y_{1}, y_{2}\right) \in \mathbb{R} \times \mathbb{R}^{N} \times \mathbb{R}^{N}$,

where $L_{0}, L_{1} \geqslant 0$ are fixed constants.

LEMMA 6.1 (Propagation of the interior ball condition) Assume that the closed set $K$ satisfies the interior ball condition with radius $r \in(0,1]$. Then the set $\mathcal{R}(t)$ satisfies the interior ball condition with radius $r e^{-\kappa t}$ for any $t \geqslant 0$, where $\kappa=3 L_{0}+L_{1}$. More precisely, if $y$ is a solution of (28) with $y(0) \in K$, if $p_{0}$ is a unit vector such that $B\left(y(0)-r p_{0}, r\right) \subset K$, and if $p:[0, T] \rightarrow \mathbb{R}^{N}$ is an absolutely continuous map satisfying

$$
\left\{\begin{array}{l}
-p^{\prime}(t)=D_{x} f(t, y(t))^{*} p(t) \\
p(0)=p_{0}
\end{array}\right.
$$

(where $D_{x} f(t, y(t))^{*}$ denotes the transpose of the matrix $D_{x} f(t, y(t))$ ), then the ball $B\left(y(t)-r e^{-\kappa t} p(t) /|p(t)|, r e^{-\kappa t}\right)$ is contained in $\mathcal{R}(t)$ for any $t \in[0, T]$.

Proof. Let $y$ and $p$ as in the lemma. Note that $p(t) \neq 0$ for any $t \in[0, T]$. 
For any $\theta \in B(0,|p(T)|)$ we consider the solution $y_{\theta}$ of the (backward) differential equation

$$
\left\{\begin{array}{l}
y_{\theta}^{\prime}(t)=f\left(t, y_{\theta}(t)\right) \\
y_{\theta}(T)=y(T)-r e^{-k T}(p(T)-\theta),
\end{array}\right.
$$

where

$$
k=2 L_{0}+L_{1} .
$$

We first prove that $y_{\theta}(0) \in K$. For this, consider the function

$$
\phi(t)=\frac{1}{2}\left|y_{\theta}(t)-y(t)\right|^{2}+r e^{-k t}\left\langle y_{\theta}(t)-y(t), p(t)\right\rangle .
$$

Note for later use that

$$
\phi(T)=\frac{1}{2} r^{2} e^{-2 k T}|p(T)-\theta|^{2}-r^{2} e^{-2 k T}\langle p(T)-\theta, p(T)\rangle \leqslant 0,
$$

since $\theta \in B(0,|p(T)|)$. Then

$$
\begin{aligned}
\phi^{\prime}(t)= & \left\langle y_{\theta}(t)-y(t), f\left(t, y_{\theta}(t)\right)-f(t, y(t))\right\rangle \\
& +r e^{-k t}\left\langle f\left(t, y_{\theta}(t)\right)-f(t, y(t)), p(t)\right\rangle \\
& -r e^{-k t}\left\langle y_{\theta}(t)-y(t), D_{x} f(t, y(t))^{*} p(t)\right\rangle \\
& -r k e^{-k t}\left\langle y_{\theta}(t)-y(t), p(t)\right\rangle .
\end{aligned}
$$

From $29($ ii)),

$$
\left\langle y_{\theta}(t)-y(t), f\left(t, y_{\theta}(t)\right)-f(t, y(t))\right\rangle \geqslant-L_{0}\left|y_{\theta}(t)-y(t)\right|^{2} .
$$

Since

$$
f\left(t, y_{\theta}(t)\right)-f(t, y(t))=\int_{0}^{1} D_{x} f\left(t, s y_{\theta}(t)+(1-s) y(t)\right)\left(y_{\theta}(t)-y(t)\right) \mathrm{d} s
$$

we have

$$
\begin{aligned}
\left\langle f\left(t, y_{\theta}(t)\right)\right. & -f(t, y(t)), p(t)\rangle-\left\langle y_{\theta}(t)-y(t), D_{x} f(t, y(t))^{*} p(t)\right\rangle \\
& =\int_{0}^{1}\left\langle\left(D_{x} f\left(t, s y_{\theta}(t)+(1-s) y(t)\right)-D_{x} f(t, y(t))\right)\left(y_{\theta}(t)-y(t)\right), p(t)\right\rangle \mathrm{d} s \\
& \geqslant-\frac{L_{1}}{2}\left|y_{\theta}(t)-y(t)\right|^{2}|p(t)|
\end{aligned}
$$

thanks to 29 (iii)). Since $|p(t)| \leqslant e^{L_{0} t}, k=2 L_{0}+L_{1}$ and $r \in(0,1]$, we have $2 L_{0}+r e^{-k t} L_{1}|p(t)|$ $\leqslant k$ for any $t \in[0, T]$. Hence we get

$$
\phi^{\prime}(t) \geqslant-k \phi(t)
$$

which gives $\phi(0) \leqslant e^{k T} \phi(T) \leqslant 0$ from 31 . Therefore

$$
\phi(0)=\frac{1}{2}\left|y_{\theta}(0)-y(0)+r p_{0}\right|^{2}-\frac{1}{2} r^{2} \leqslant 0,
$$

which proves that $y_{\theta}(0) \in K$ because $B\left(y_{0}-r p_{0}, r\right) \subset K$.

Since $y_{\theta}(0) \in K$, we also have $y_{\theta}(T)=y(T)-r e^{-k T}(p(T)-\theta) \in \mathcal{R}(T)$ for any $\theta \in B(0,|p(T)|)$. Hence $\mathcal{R}(T)$ satisfies the interior ball condition with radius $r e^{-k T}|p(T)|$. Since $|p(T)| \geqslant e^{-L_{0} T}$, we have finally proved our claim with $\kappa=k+L_{0}=3 L_{0}+L_{1}$. 


\section{Acknowledgements}

The authors would like to thank G. Barles, O. Ley and P. Cannarsa for fruitful discussions during the preparation of this article. This work was supported by the two contracts ACI JC 1025 (2003-2005) and ACI JC 1041 (2002-2004).

\section{REFERENCES}

1. Alibaud, N. Existence and uniqueness for non-linear parabolic equations with non-local terms. Preprint (2004).

2. Alvarez, O., Hoch, P., Le Bouar, Y., \& Monneau, R. Résolution en temps court d'une équation de Hamilton-Jacobi non locale décrivant la dynamique d'une dislocation. C. R. Acad. Sci. Paris Sér. I 338 (2004), 679-684. Zbl 1049.35005 MR 2065373

3. Alvarez, O., Hoch, P., Le Bouar, Y., \& Monneau, R. Dislocation dynamics: short time existence and uniqueness of the solution. Arch. Rat. Mech. Anal., to appear.

4. Ambrosio, L., Fusco, N., \& Pallara, D., Functions of Bounded Variation and Free Discontinuity Problems. Oxford Math. Monographs, Clarendon Press, Oxford (2000). Zbl 0957.49001 MR 1857292

5. BARdi, M. \& CAPuzzo-DolcetTA, I., Optimal Control and Viscosity Solutions of Hamilton-JacobiBellman Equations. Birkhäuser, Boston (1997). Zbl 0890.49011 MR 1484411

6. Barles, G. Solutions de Viscosité des Equations de Hamilton-Jacobi. Springer, Berlin (1994). Zbl 0819.35002 MR 1613876

7. Barles, G., Soner, H. M., \& Souganidis, P. E. Front propagation and phase field theory. SIAM J. Control Optim. 31 (1993), 439-469. Zbl 0785.35049 MR 1205984

8. Cannarsa, P. \& Cardaliaguet, P. Perimeter estimates for the reachable set of control problems. $J$. Convex Analysis, to appear.

9. CANnARSA, P. \& FrankowsKa, H. Interior sphere property of attainable sets and time optimal control. Preprint (2004).

10. Cannarsa, P. \& Sinestrari, C. Semiconcave Functions, Hamilton-Jacobi Equations and Optimal Control. Birkhäuser, Boston (2004). Z Zbl pre02129788 MR 2041617

11. Cardaliaguet, P., On front propagation problems with nonlocal terms. Adv. Differential Equations 5 (2000), 213-268. Zbl 1029.53081 MR 1734542

12. CARoff, N. \& FrankowsKa, H. Conjugate points and shocks in nonlinear optimal control. Trans. Amer. Math. Soc. 348 (1996), 3133-3153. Zbl 0924.49016 MR 1344204

13. Clarke, F. The maximum principle under minimal hypotheses. SIAM J. Control and Optim. 14 (1976), 1078-1091. Zbl 0344.49009 MR 0415453

14. Evans, L. C. \& Gariepy, R. F., Measure Theory and Fine Properties of Functions. Stud. Adv. Math., CRC Press, Boca Raton, FL (1992). Zbl 0804.28001 MR 1158660

15. Federer, H. Geometric Measure Theory, Springer (1969). Zbl 0176.00801 MR 0257325

16. Hirth, J. R. \& Lothe, L. Theory of Dislocations. 2nd ed., Krieger, Malabar, FL (1992).

17. Kubin, L. P., Canova, G., Condat, M., Devincre, B., Pontikis, V., \& Bréchet, Y. Dislocation microstructures and plastic flow: a 3D simulation. Solid State Phenomena 23 \& 24 (1992), 455.

18. LARDNER, R. W. Mathematical Theory of Dislocations and Fracture. Mathematical Expositions 17, Univ. of Toronto Press (1974).

19. Lorenz, T. Boundary regularity of reachable sets of control systems. Systems Control Lett. 54 (2005), 919-924.

20. NABARro, F. R. N. Theory of Crystal Dislocations. Clarendon Press, Oxford (1969).

21. Rodney, D., Le Bouar, Y., \& Finel, A. Phase field methods and dislocations, Acta Mater. 51 (2003), $17-30$. 